\title{
Interpretivist Tradition in Qualitative Anthropological \\ Research Writings
}

L Prakash Upadhyay, PhD

\section{Abstract}

Stranded in a philosophical position, qualitative research is fundamentally "Interpretivist" in the sense that it is concerned with how the social world is realized, interpreted, understood and experienced, or produced. Qualitative investigation based researches rummage around for answers to their questions in the realistic world. They congregate what they see, hear and read from the people and places and from events and activities and their main purposes are to learn about some aspects of the social world and to generate new understandings that can be used by that social world. Anthropological qualitative research is about the interpretation of social world especially of cultures and people's life-ways rather than seeking causal explanations for cultural practices. In anthropological writings as much attempt and consideration should be given to the organization of the scientific paper and article as was given to the execution of the anthropological study, the writer should employ assorted techniques to make the belief, faith based qualitative anthropological study more and more empirical.

Keywords: Interpretivist, transcripts, recursive, holographic, redundancy, histograms

Cultures are prototype of human behavior and knowledge that every human discovers as a member of a society and anthropology is the study of human being in the changing context. Performing everything that engrosses comprehending human cultural, social, economic, political and other behaviors which are intangible and qualitative are the ingredients of anthropological studies which are primarily qualitative and hence anthropologists are the trained observers who collect factual empirical information about people by watching, listening and interpreting what others are doing at micro level at the plebs level. Allied candidly with people's know-how, outlooks, and interpretations of the situations, qualitative data is unique as it is extracted from the heart and mind of the people. 


\section{Interpretivist Tradition in Qualitative Research: Archetype of ethnographic work in Nepal}

Interpretation is the major building block of qualitative research which is unswervingly related to people's experiences. Barnard, Andy, Burgess, Terry and Kirby, Mike (2004) articulates that qualitative data deals directly with people's experiences. It is grounded in a philosophical position, which is broadly 'Interpretivist' in the sense that it is concerned withhow the social world is interpreted, understood and experienced, or produced. In anthropology, qualitative researchers seek answers to their questions in the real world. They gather what they realize, see, hear and read from the people and places and from events and activities and the main intentions are to learn about some aspects of the social world and to engender new understandings that can be used by that social world.

Concepts, theoretical perspectives, methods, tools and techniques differ sharply in qualitative and quantitative studies. Quantitative and the qualitative approaches have their flaw as well as potency. Both qualitative and quantitative classifications are based on three groupings: study purpose, measurements of variables and the analysis of information

The study is classified as qualitative if the purpose of the study is primarily to describe a situation, phenomenon, problem or event, the information is gathered through the use of variables measured on nominal scale (good, better, satisfactory, poor, etc.), or ordinal scale (first, second, third, etc.) i.e. qualitative measurement scales; if the analysis is done to establish the variation in the situation, phenomenon or problem without quantifying it, e.g. the description of an observed situation, the historical enumeration of events, and account of different opinions people have about an issue.

Methods selection with their tools and techniques depends upon the main purpose of research, discipline and theoretical stance. Both qualitative and quantitative methods observe different realities or different aspects of reality; nevertheless the combination of these two methods will lead to a very balanced and high quality research. The differences between qualitative and quantitative are immense. For example, qualitative methods are less objective as they rely on qualitative data, a scenario is based on natural settings with search for meaning, rejection of natural science; stand on idealist perspective with identification of cultural patterns. Qualitative methods are based on Inductive Approaches. On the 
other hand, quantitative methods in sociology and other discipline are more empirical as they are based on statistical data with the provision of experimental settings, adoption of natural science, pursuance of scientific laws with realist perspective and deductive approaches.

Interpretivist tradition of qualitative method in anthropology deals with the systematic approach signifying the art of asking, listening, and interpreting. The methods of collecting qualitative data can be grouped into four consistent groupings. The methods, researcher's role, techniques and study site tradition are:

\begin{tabular}{|lllll|}
\hline Methods & Researcher's role & Technique of data collection & & Study site tradition \\
Observation & Observer & Participant observation & & Field work tradition \\
In-depth interview & Interviewer & In-depth (key informant interview) & Field work tradition \\
Group discussion & Group moderator & Focus group discussion & Field work tradition \\
\hline
\end{tabular}

The interpretivist tradition's chief dictum of qualitative methodology is to get people to open up and let them express themselves in their own terms and at their own pace in their own way. The prime issue is going among the people and building rapport for conducting a lofty research. Developing rapport is establishing harmonious relationship, togetherness, agreement, affinity, sympathy, concord empathy. It means winning the heart and mind of the people on whom the researcher's research is targeted. It is done through rigorous and intensive field work tradition. Ethnographic researches are the excellent example of qualitative method. Sherpas through their Rituals (1978) is a qualitative ethnographic works in/on Nepal by Sherry Ortner. Ortner conducted her rigorous field study in Dzemu 'Solu' from September 1966 to February 1968. She collected primary data through field work through methods like ethnography, group discussion, interview, and observation. Her theoretical base was ritualistic approach.

Ortner hasused an assortment of diverse anthropological approaches that she exposed during her research and put into practice in her fieldwork and analysis. Although many approaches are used, of particular importance to her ethnography are the theoretical approaches of structuralism and symbolic analysis in a qualitative manner, which although used particularly in interpretive approaches has been applied to various theoretical frameworks. This tendency towards symbolic analysis is evident by Ortner's stated view that culture is always biased, selected, partial and interpreted. This is also evident in the use of semantic (meanings and 
words of sentence) structure analysis and the focus on symbols as a way of imposing meaning. The theoretical approach that is the focus of analysis is structuralism; in particular this close scrutiny is achieved by focusing on the exemplary paradigmatic set and the role of ritual as a mediator and reconciler between contrasting aspects of society. By giving fastidious attention to certain similarities that bear a resemblance to each other within mysterious religious rites and symbolism portrayed in the ethnography it is probable to visibly interpret and understand the way that structural analysis can, and has indeed been employed to access the communicative code of the Sherpa society. While interpreting and analyzing the symbols of Sherpa rituals, Sherry Ortner leads us toward the detection of conflict, contradiction, and stresses in the wider social and cultural world--- the best examples of interpretivist tradition in anthropological ethnographic study. Following a general ethnographic sketch a brief description and interpretation of a ritual has been made by Ortner. The ritual has been sliced up, and its symbolic elements are used as guides in the exploration and interpretation of problematic structures, relationships, and ideas of the Sherpa culture. Nyungne, exorcisms and offering rituals have been analyzed and interpreted by Ortner and are the case of hospitality (secular event) in order to present the network of Sherpa culture.

\section{Qualitative Data Analysis (QDA) Process in Anthropology}

Anthropology is about the interpretation of cultures and people's life-ways rather than seeking causal explanations for cultural practices. American anthropologist Clifford Geertz was a foremost figure of the interpretive or the symbolic anthropology school. According to the interpretive approach in anthropology, meaning is constituted out of interaction between individuals; the symbols and meanings are public in their significance and are not confined to single individual brains or the private sphere. It is the public or social aspect of meaning that is the focus of analysis for the proponents and followers of this approach. Geertz makes an influential case for anthropology as an interpretive science by clarifying that clarification of details in local cultural context rather than aiming at grand comparison is "interpretive anthropology" and also the way for anthropological work. For Geertz the concept of culture is fundamentally a semiotic one and he makes it clear in one of his most frequently quoted statements (1973):

Believing with Max Weber that man is an animal suspended in webs of significance he himself has spun, I take culture to 
be those webs, and the analysis of it to be therefore not an experimental science in search of law but an interpretive one in search of meaning.

Hence, interpretive approach discards attempts at causal explanations for cultural phenomena. Geertz also used the metaphor of thick description which means the interpretation of all the minor details of human life in thick layers of contextual significance. According to Clifford Geertz "ethnography is thick description" and therefore his approach to culture is based on the metaphor of culture as text. Given this, an ethnographer's role is to interpret the text in a qualitative world - that is, reading cultures as if they were texts of some sort. The Gurung Arghau or Pae and the Ghapren (Gurung priest) reciting the mantras may be an illustration.

Based on above argument I argue that qualitative analysis understands phenomenon and to go beyond reporting - move towards interpretation and identify themes and sub-themes. In the process of research, collection and analysis occur simultaneously and human-as-an-instrument is the innermost point. Results can be used to investigate for further information and detail but the risk remains of diverting from research objectives. Nevertheless qualitative data analysis analyzes text, interview transcripts, photographs, art, field notes of observations, etc. During the whole process of qualitative data analysis, it is an admirable idea to engage in recording reflective notes about what we are learning from our data. The idea is to write memos (notes) when we have ideas and insights and to include those memos as additional data to be analyzed.

Several phases can be distinguished in qualitative data analysis process:

Data cleaning

Initial data analysis (assessment of data quality)

Main data analysis (answer the original research question)

- Final data analysis (necessary additional analyses and report)

Basic processes in QDA serve a process of noticing, collecting and thinking. There is a simple foundation to the complex and rigorous practice of QDA. Once we take hold of this foundation we can move about in many different directions. The consequence is the idea that Qualitative Data Analysis (QDA) is a masterpiece based on three notes: Noticing, Collecting, and Thinking about interesting things. 
Th irrik adbut thires:
Collect Lfoitres:

Source: Seidel, 1998

Qualitative data analysis process is not linear. When we do qualitative data analysis we do not simply Notice, Collect, and then Think about things, and then write a paper or report, rather, the process has the following characteristics (Seidel, 1998):

a) Iterative and Progressive: The process is iterative and progressive as it is a cycle that keeps repeating. For example, when we are thinking about things we also start noticing new things in the data. We then collect and think about these new things. In principle the process is a never-ending twist (spiral).

b) Recursive: The process is recursive because one part can call us back to a previous part. For example, while we are busy collecting things we might simultaneously start noticing new things to collect.

c) Holographic: The process is holographic in that each step in the process contains the entire process. For example, when we first notice things we are mentally collecting and thinking about those things. Hence, while there is a simple foundation for qualitative data analysis, the process of doing qualitative data analysis is complex. The key is to root researcher in this foundation and the rest will flow from this foundation.

Order in Paradox: Myth, Ritual, and Exchange among Nepal's Tamang (1989) by David Holmberg incorporates -iterative and progressive, recursive, holographic characteristics. Holmberg conducted field work and his analysis is based on noticing, collecting and thinking. Holmberg's analysis is not only an ethnographic account but penetrates the Shaman/ Sorcery Buddhism and traditions of sacrifice based complex cultural structure of the Tamangs. The analysis process is holographic in that each step in the process contains the entire process. The focus beyond the ethnographic is on the structure of a complex ritual field composed of Buddhist, Shamanic, and sacrificial practitioners and on the relation of this structure to the logics and contradictions of Tamang social exchanges, 
especially those associated with cross-cousin marriage. The role of symbolism remains vital in Tamang culture. The author says;

Tamang symbology was characterized by a mythic closure which is related directly to a highly localized geography, society, polity, and economy. The world of Tamdungsa - the Tamang village where I resided-has now opened up.

Holmberg squabbles that the political symbology which gave the local ritual substance has been translated in large measure into an idiom of kinship where kinship and polity are no longer coextensive as they have been in the past.

\section{Qualitative Scientific Paper and Research Article Writing in Anthropology}

Anthropology is related with the certification and rationalization of similarities and differences, constancy and alteration in human behavior. Numerous epistemological and perspectives in anthropology are alleged to be more appropriate than the others by the supporters of each of such perspectives for advancing our understanding of the society, culture as well as human nature, behavior and activities. However, statements and arguments or explanations ought to rely on the observable reality or social facts. Differences of viewpoints are perceptible even in defining what consists of the observable reality or facts. The realities or facts defined by the supporters of the varying theoretical perspectives make us believe that observable reality or the facts are relative things rather than consisting of absolute nature as they change according to the theoretical stance. Given this, while conducting qualitative anthropological researches, anthropologists need to contemplate on theoretical stance. While writing anthropological research report, or scientific paper of any sort, they ought to pay more attention to observable reality or social facts. Side by side it is vital to note that qualitative researches are usually complicated hence writing scientific paper and research article based on qualitative methods is a difficult task as scientific research demands precision but qualitative approaches are based more on fewer objectives and more idealist notion (Upadhyay, 2011) and are far from observable reality. Nevertheless, it is necessary to write papers with precision, clarity and economy. In a scientific paper and research article each and every judgment should convey the exact truth as simply as possible by reflecting precision in the form of clarity. Regrettably, a glimpse of any scientific journal will make 
known that the above-stated ideals of precision, clarity, economy, etc. is often not attained in the real world of scholarly publication. Allegation of gloominess and elitism within the scientific community probably originate in the sad fact that many research scholars are not capable of expressing their hypotheses and conclusions clearly and simply. Nevertheless, much of the uncertainty can be eliminated if writing is considered part of the self-importance. It means that as much attempt and consideration should be given to the organization of the scientific paper as was given to the execution of the study, and that the writer should employ easy and digestible sentences not jumbled with excess verbiage.

A great deal is spent to perform research---money, resources and hard work, and the publication is the essence of that expensive work, all that will survive and communicate what was learned. In many cases it is difficult to get a scientific paper published in reputed journal. In many cases the researchers will have to pay to have papers published in reputed journals. If the content is not clear, the research will be lost, and the money spent will be wasted. If the text is vociferous, talkative and over descriptive the readers will be bored to read and the status of the whole scientific paper will ultimately shrink.

The particular format and style adopted for a qualitative scientific paper and research article depend on both the nature of the report and the journal or other publication in which the paper is to be published. It means there is often more than one acceptable way of doing something, depending on author's intentions. Authors require to become familiar with details of organization, section headings, methods of data presentation, and ways of citing and listing references by examining recent papers in any wellestablished journal.

\section{Sketch of Qualitative Scientific Paper and Research Article in Anthropology}

The scientific paper and research article may have the following elements: Title, Abstract, Introduction, Objectives and Methods, Domino effect or Results, Discussion, Conclusion, Literature Cited (References)

The words 'Introduction', 'Methods,' etc. are used to head the sections of researcher papers, each section beginng on a new page. Tables and figures are placed at the end of the text. In the Appendix, the researcher will present the elements described, except for an abstract.

\section{Title}

The title should contain these elements: The catchy name of the topic 
studied; the particular aspect studied; the variable(s) manipulated.

It is necessary that grammatical creativeness and clarity of language should be maintained thoroughly.

\section{Abstract}

The abstract should be one or two paragraph condensation (150-200 words) of the whole work described completely in the article. The abstract should be a self-contained unit capable of being understood without the benefit of the text. It should contain these four elements:

1. the purpose of the study (the central question);

2. a brief statement of what was done (Methods);

3. a brief statement of what was found (Results);

4. a brief statement of what was concluded (Discussion, in part).

\section{Introduction}

Introduction is the background of the work. It deals with the topic in general and how the researcher developed interest in the research topic. It provides necessary background and setting to put problem in proper context and fixing basis for study and sets the stage for the research and puts the topic in perspective. In general, the function of an introduction is to present the question being asked and place it in the context of what is already known about the topic. Background information that suggests why the topic is of interest and related findings by other scientists are usually mentioned here. In other words, this introduction section should include:

1. A description of the nature of the problem and current state of knowledge or understanding at the beginning of the investigation (background);

2. A statement of the purpose, scope, and general method of investigation in the study;

3. Hypothesis/hypotheses and predictions.

However there are few tips to keep in mind while writing introduction. For example, keep in head that do not get lost in reviewing background information. Remember that the Introduction is intended to introduce the reader to your research, not to summarize or evaluate all past literature on the subject (which is the purpose of a review paper). You may be attracted to discuss numerous other concepts and ideas in your Introduction section, but they are better saved for the Discussion section, where they become a powerful tool for comparing and interpreting the results. 
You should remember that you should take account of only adequate background information to enable the readers to understand why you are asking the questions and why your hypotheses are reasonable ones. Normally, a short explanation of the theory involved is sufficient. The statement of purpose expresses the central question you are asking and hence present the variable you are investigating.

Some scholars believe that the principal results and conclusions should be summarized in the Introduction section. However, the majority of scholars disagree, arguing that such a summary appears in the abstract and should not be repeated in the Introduction. You should stay away from such practice except when writing for a journal that requires it. Introduction $i$ is written in the past or present tense, never in the future. You should avoid expressions like 'This study will examine'.

\section{Objective and Method}

The section related to objective and methods is mainly related to the objectives of the research study and methodological issues. The function of this section is to describe purpose and all investigational procedures, including the relationships between variables. The explanation should be complete enough to enable someone else to repeat researchers work. It is up to researcher to decide what order of presentation will make the most sense to readers.

Method sections comprises of the entire research methodology and procedure adopted for research. In social sciences the paper may spell out the location of the site/place where the author has done the research with clear reasons for selection of the site/place. It should mention sampling frame by delineating the population or the universe of the study. It should describe the size of the sample rationale for the size and its determination, sampling units, a procedure to ascertain a reliable and adequate sampling frame and the sources of data. Authors should mention the basic design of data collection according to the type of study, and how they collected the data. Likewise, it is furthermore crucial to pay attention to undermentioned points:

1. Explain why each procedure was followed, i.e., what variable were you measuring and why?

2. Research procedures and results are narrated in the past tense (what you did, what you found, etc.) whereas conclusions from your results are given in the present tense. 
3. Mathematical equations and statistical tests are considered mathematical methods and should be described in this section along with the actual research based investigational work.

4. Use active rather than passive voice when possible. Always use the singular " $\boldsymbol{I}$ " rather than the plural "we" when you are the only author of the paper. Throughout the paper, avoid contractions, e.g. did not vs. didn't.

5. If any of your methods is fully described in a previous publication (yours or someone else's), you can cite that instead of describing the procedure again.

\section{Results}

Result section is one of the major sections in scientific paper and article. But in some journals, Results and Discussions are joined in a single section so as to permit a single integrated treatment with minimum repetition. This is more appropriate for short, simple articles than for longer, more complex ones.

The purpose of Result section is to summarize general trends in the data without comment, bias, or interpretation. Statistical tests applied to your data are reported in this section although conclusions about your original hypotheses are saved for the Discussion section. Data may be presented in figures and tables, but this may not substitute for a verbal summary of the findings. The text should be understandable by someone who has not seen your figures and tables.

1. All results should be presented, including those that do not support the hypothesis.

2. Statements made in the text must be supported by the results contained in figures and tables.

3. The results of statistical tests can be presented in parentheses following a verbal description.

\section{Discussion}

This section of a paper/article is related to the discussion of data. The function of this section is to analyze the data and relate them to other studies. To analyze means to evaluate the meaning of your results in terms of the original question or hypothesis and point out their significance. It ought to be noted that the Discussion section should contain at least:

- The relationship between the results and the original hypothesis, i.e., 
whether they support the hypothesis, or cause it to be rejected or modified.

- An integration of your results with those of previous studies in order to arrive at explanations for the observed phenomena.

- Possible explanations for unexpected results and observations, phrased as hypotheses that can be tested.

- Realistic investigational procedures, which the author should describe.

- Trends that are not statistically significant can still be discussed if they are suggestive or interesting, but cannot be made the basis for conclusions as if they were significant.

Discussion is a vital section of scientific the paper/article; hence it should:

- Avoid redundancy between the Results and the Discussion section. Do not repeat detailed descriptions of the data and results in the Discussion.

\section{Conclusion}

The conclusion of the study should be properly reached. It is the winding up or the hidden treasure of the study and hence should present the finale of the findings in a very succinct and laconic way.

The Discussion ends (concludes) with a summary of the principal points you want the reader to remember. This is also an appropriate place to propose specific further study if that will serve some purpose, but do not end with the tired cliché' that 'this problem needs more study.' All problems need more study. Do not close on what you wish you had done; rather finish stating your conclusions and contributions.

\section{Literature Cited (References)}

Literature cited or reference is the last section. References are listed by author, as indicated by the following sample list. Papers are not referred to by footnotes as in literature papers but are cited within the body of the text. The format of scientific paper and article also differs according to the types of journals publishing the paper; hence it is necessary to follow the specific format they want author to adopt for the work. Remember that in the case of more than three authors, author may use et al. (from "et alii," Latin for "and others") after the first author's name. No reference is listed in this section unless it was cited somewhere in the text and plagiarism must be strictly avoided as it is unethical and an offense.

\section{- Use of Tables and Figures in the paper/article}


Tables and Figures ought to be used when they are a more efficient way to convey information than verbal description. They must be independent units, accompanied by explanatory captions that allow them to be understood by someone who has not read the text. Do not repeat in the text the information in tables and Figures, but do cite them, with a summary statement when that is appropriate. Whenever possible, use a Figure instead of a table. Relationships between numbers are more readily take hold (grasped) of when they are presented graphically rather than as columns in a table. Nevertheless there are certain tips related to tables and figures which need to be remembered viz.

\section{Tables}

- It is necessary that you do not repeat information in a table that you are depicting in a graph or histogram; include a table only if it presents new information.

- It is easier to compare numbers by reading down a column rather than across a row. Therefore, list sets off data you want your reader to compare in vertical form.

- Provide each table with a number (Table 1, Table 2, etc.) and a title. The numbered title is placed above the table.

\section{Figures}

- Figures comprise graphs, histograms, and illustrations, both drawings and photographs. Provide each Figure with a number (Fig. 1, Fig. 2, etc.) and a caption that explains what the Figure shows. The numbered caption is usually placed below the Figure.

- Remember that the Figures submitted for publication must be ready made, i.e., they will appear just as you submit them, or photographically reduced. Lines should be drawn with black ink (not ballpoint or marker). Symbols, letters, and numerals must be produced by stencil or mechanically, and should be large enough to withstand reduction. Proportions must be the same as those of the page in the journal to which the paper will be submitted.

\section{- Graphs and Histograms}

Both graphs and histograms can be used to compare two variables. Nevertheless, graphs show continuous change, whereas histograms show discrete variables only. Compare Figures in the Appendix. You can compare groups of data by plotting two or even three lines on one graph, but avoid cluttered graphs that are hard to read, and do not plot unrelated 
trends on the same graph. For both graphs, and histograms, plot the independent variable on the horizontal (x) axis and the dependent variable on the vertical (y) axis. Label both axes, including units of measurement.

\section{- Drawings and Photographs}

These are used to illustrate the events, investigational apparatus, models, etc, and results of procedures. Putting in order such Figures is a lot of work and can be tiresome and expensive too, so each Figure must add enough to justify its preparation and publication, but good Figures can significantly improve the quality of a professional article.

\section{Insinuation}

Anthropology is an altering science. It is no longer the study of preliterate people. Rather it is the study of modern people with both rural and urban backgrounds. Qualitative research is the hallmark of anthropology and anthropological researches. Media revolution has revolutionized anthropology and anthropological researches. Expansion in mass communication, telecommunication, electronic media like frequency module (F.M.) radio television, media, and information technology have marvelous insinuation for anthropology-its concepts, theory, methods, field study/stay, analysis, practice, report writing, etc. Interpretivist tradition in qualitative anthropological research is undergoing the alteration concerned with interpretation of social world, understanding of social world and experiences of social world. Anthropologists seeing, hearing and reading from the people and places, events and activities are altering. Their learning about aspects of the social world for generating new understandings that can be used by that social world is under modification. Similarly the studied group may now be conversing, discoursing, raising questions, making remarks, doing arguments, giving and sharing opinions with logics with qualitative researcher straightforwardly or obliquely. Given this, the vital concern now is how the existing anthropological concepts, theories and methods are going to concentrate on different problems and issues appropriately and sufficiently in order to get an evocative and holistic image - which was once a hallmark of anthropology and is still prevailing. Nevertheless in the very speedily changing world, innovative traditions of the perception of emerging contexts and realities need to be exposed and accepted by anthropologists and need to be practiced in anthropological studies and writings. 


\section{References}

Barnard, Andy, Burgess, Terry and Kirby, Mike (2004). Sociology. U.K. Cambridge University Press.

Geertz, Clifford, 1973. "Thick Description: Toward an Interpretive Theory of Culture." Chapter 1 in The Interpretation of Cultures (pp. 3-30). New York: Basic Books.

Holmberg, David, 1989. Order in Paradox: Myth, Ritual, and Exchange among Nepal's Tamang. Ithaca and London: Cornell University Press.

Ortner, Sherry B. (1978). Sherpas Through their Rituals. New York: Cambridge University Press.

Ortner, Sherry B. (1984) Theory in Anthropology since the Sixties, Comparative Studies in Society and History. 26: 126-166.

Seidel, John (1998). 'Method and Madness in the Application of Computer Technology to Qualitative Data Analysis.' In N. Fielding and R. Lee, (Eds.) Using Computers in Qualitative Research. Newbury Park: Sage Publication.

Upadhyay, P (2011). Research Methods in Sociology/Anthropology. Kathmandu, Khitiz Publication. 